\title{
On the State of Stress in the Growth Plate under Physiologic Compressive Loading
}

\author{
Jie Gao, John L. Williams, Esra Roan* \\ Department of Mechanical Engineering, Biomedical Engineering, The University of Memphis, Memphis, USA \\ Email: "eroan@memphis.edu
}

Received November 19, 2013; revised December 19, 2013; accepted December 26, 2013

Copyright (C) 2014 Jie Gao et al. This is an open access article distributed under the Creative Commons Attribution License, which permits unrestricted use, distribution, and reproduction in any medium, provided the original work is properly cited. In accordance of the Creative Commons Attribution License all Copyrights (C) 2014 are reserved for SCIRP and the owner of the intellectual property Jie Gao et al. All Copyright (C) 2014 are guarded by law and by SCIRP as a guardian.

\section{ABSTRACT}

The growth plate is a thin layer of cartilage sandwiched between epiphyseal and metaphyseal bone and is the location of active bone growth during childhood. It is subjected to large compressive and shear forces while protecting its resident chondrocytes from damage. We believe that computational modeling can help us better understand how the macro-scale loads are transmitted to micro-scale stresses and strains within the growth plate cartilage. As a first step in this process we analyzed the mechanical response of compression experiments performed on bovine bone/growth plate/bone samples. We endeavored to estimate the modulus of elasticity of the growth plate itself by simulating the compression experiments of these specimens using the finite element method. It is shown that when the growth plate in the compression specimens was modeled as a flat layer, the state of stress in the cartilage was triaxial and non-uniform with the hydrostatic stress being much greater than the octahedral shear stress over most of the central region of the growth plate test samples. The computational models accounted for variations in the average cartilage thickness, the non-uniaxial, non-uniform and triaxial state of stress in the thin cartilage layer, and for the estimated extrinsic compliance resulting from compression of the variable heights of bone on either side of the growth plate cartilage. However, due to lack of information on the internal structure of each sample, the models did not account for the variations in the non-flat topography of the growth plates. The models also did not include the calcified cartilage layer. Further model development is recommended in order to determine the degree to which accounting for the complex growth plate topography influences the predicted cartilage modulus of elasticity.

\section{KEYWORDS}

Growth Plate; Physis; Cartilage; Mechanical Properties; Finite Element Analysis

\section{Introduction}

The growth plate, a cartilage plate separating the metaphysis from the epiphysis at each end of a long bone (Figure 1A), is responsible for the longitudinal growth of bone. It consists of chondrocytes embedded in an abundant extracellular matrix (ECM) [1]. The growth plate is composed of a cartilaginous component that has three histologically distinct zones: reserve or germinal, proliferative and hypertrophic [2] (Figure 1B). The longitudinal growth of bones is controlled by the rate of chondrocytic proliferation and the amount of chondrocytic en-

\footnotetext{
"Corresponding author.
}

largement (hypertrophy) in the growth direction [3]. From the epiphyseal side nutrients are provided by the epiphyseal blood vessels passing through the germinal zone and terminating at the proliferating layer and then progress through the growth plate via diffusion [4]. On the metaphyseal side vascular loops penetrate into the spaces of dying hypertrophic chondrocytes to provide nutrients for the osteoprogenitor cells producing bone on the primary spongiosa cartilage scaffolds.

The existence of chondrocytes in a soft collagen rich ECM nestled between calcified bones and subjected to significant mechanical loads renders the growth plate to be a mechanobiological structure, which is highly sensitive 


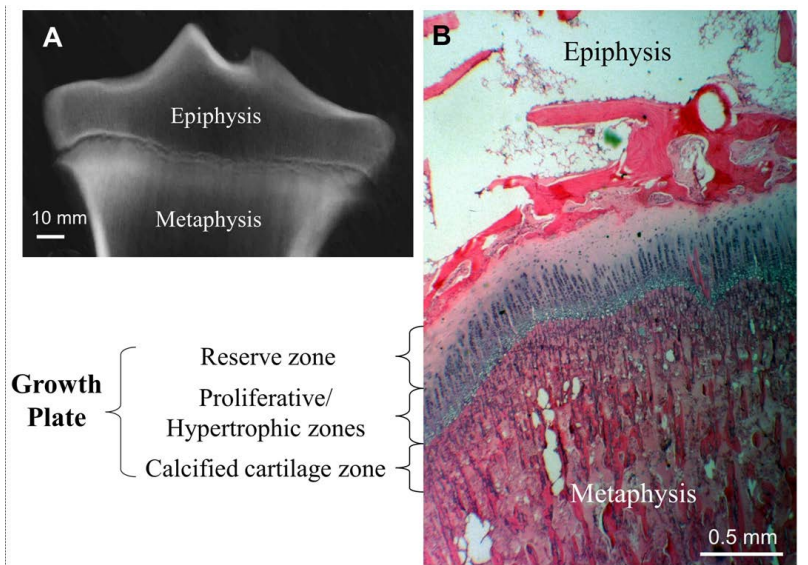

Figure 1. (A) Radiograph of a 1-inch coronal slice through the proximal tibial growth plate of a 5 month-old calf. Primary mamillary processes can be seen towards the left and right borders with the secondary mamillary processes just barely visible; (B) Histological zones of the proximal tibial growth plate of a 4 - 5 month-old calf.

to mechanical factors as well as biochemical signals. Broad laws have been proposed to govern bone modeling and remodeling, and longitudinal growth, such as Wolff's law and Hueter-Volkmann law [5,6], respectively. Wolff's law states that bone grows and remodels in response to the forces that are placed upon it. Hueter-Volkmann law states that increased pressure acting on the growth plate retards bone growth and conversely, reduced pressure or even accelerates its tension [3,5,6]. Frost proposed that for stresses not exceeding the physiological range, endochondral bone growth speeds up in the case of compression compared to tension, and that compression exceeding physiological range slows down or even inhibits growth [7]. It has further been proposed in engineering mechanics language similar to that used to describe failure theories, that hydrostatic pressure maintains cartilage while octahedral shear stress promotes its degradation and ossification $[8,9]$.

Although discrepancies exist between these fundamental laws, it is clear that mechanical loading can modulate bone growth. This phenomenon has key implications in infant and juvenile pathological progressive musculoskeletal deformities, such as idiopathic scoliosis, bowlegs and others $[10,11]$. Meanwhile, although physiologic levels of compression are essential for bone development, excessive compressive loading may damage the physeal cells in the germinal and proliferative zones and lead to bone growth retardation or cessation causing such abnormalities such as late-onset tibia vara (Blount's disease) [12]. Clinical treatment of these deformities is often directed at modifying the mechanical environment of the affected bone [1]. However, the compressive injury of the growth plate is clinically invisible and not easily diagnosed at the time of injury and the underlying me- chanisms of this type of injury still remain unknown. Despite many studies, our quantitative and physiological understanding of how bone growth is regulated in response to mechanical loading is still limited [1]. Computational models of the growth plate under compression may yield insights into the micro-mechanical environment of the cells in the growth plate, which may help guide diagnoses and develop treatments in the future.

Several studies have reported the compressive mechanical properties of growth plate under different loading conditions using different animal models (Table 1). In this work, we analyzed the mechanical response of macroscopic bovine growth plate samples in uniaxial compression. The aim of this study was to use finite element (FE) analyses to obtain the inherent compressive mechanical properties of the growth plate assuming the cartilage tissue to be homogeneous and isotropic and linearly elastic and to explore the state of stress within this thin cartilage layer. We view this macroscopic model as a first in a series of steps needed to construct a more complete microscopic FE model of the growth plate structure including zonal and cellular details.

\section{Materials and Methods}

\subsection{Previous Uniaxial Compression Experiments of Macroscopic Samples}

Finite element models were developed to simulate uniaxial compression experiments that were previously conducted on bone-growth plate-bone samples prepared from three fresh-frozen 5-month-old calf proximal tibial growth plates [13]. The specimens were cut from four sites in each growth plate: antero-lateral and -medial and postero-lateral and -medial. All $7 \times 7 \times$ height mm block samples were prepared so as to maintain the minimum height without encroaching on the growth plate cartilage whilst keeping the orientation aligned with the tibial longitudinal axis. Samples were immersed in protease inhibitor solution and frozen until testing.

Bone/growth plate/bone specimens were first preloaded to $1 \mathrm{~N}$ and then compressed at $0.055 \mathrm{~mm} / \mathrm{min}$ to a gripto-grip strain of $20 \%$ and held at this strain until $1400 \mathrm{sec}$ had elapsed and complete stress relaxation had occurred (Figure 2). A schematic structure of sample geometry is shown in Figure 3A. The equilibrium modulus, which can be considered as the elastic modulus at $20 \%$ strain level, was calculated for each specimen by dividing the equilibrium stress by the grip-to-grip strain and reported previously [13]. The slopes of these twelve stress-strain curves, which reflect the extrinsic elastic moduli of these samples $\left(E_{E X}\right)$, were obtained by curve fitting a straight line to the data between 0 and $20 \%$ strain using Matlab (Natick, MA). 
Table 1. Material properties of the growth plate obtained from various experimental modalities.

\begin{tabular}{|c|c|c|c|c|c|c|}
\hline Studies & Species & Age & Location & Method & Material type & Material properties \\
\hline $\begin{array}{l}\text { Sergerie, } \\
\text { et al. (2009) } \\
\quad[16]\end{array}$ & swine & newborn & $\begin{array}{l}\text { distal } \\
\text { ulnae }\end{array}$ & $\begin{array}{l}\text { unconfined compression } \\
\text { stress } \\
\text { relaxation tests }\end{array}$ & $\begin{array}{l}\text { transversely } \\
\text { isotropic } \\
\text { biphasic }\end{array}$ & $\begin{array}{l}\mathrm{E}_{3}=0.51 \pm 0.12 \mathrm{MPa}, \mathrm{E}_{1}=8.65 \pm 1.72 \mathrm{MPa} \\
\mathrm{v}_{21}=0.24 \pm 0.07, \mathrm{v}_{31}=0.08 \pm 0.03 \\
\mathrm{k}_{1}=1.82 \pm 0.67 \times 10^{-15} \mathrm{~m}^{4} / \text { Ns equilibrium state }\end{array}$ \\
\hline $\begin{array}{l}\text { Barthelat, } \\
\text { et al. (1999) } \\
\text { [24] }\end{array}$ & rabbit & 1, 3, 8 weeks & $\begin{array}{l}\text { proximal tibia, } \\
\text { distal ulna, distal } \\
\text { radius }\end{array}$ & $\begin{array}{l}\text { unconfined compression } \\
\text { stress relaxation tests }\end{array}$ & $\begin{array}{l}\text { transversely } \\
\text { isotropic } \\
\text { biphasic }\end{array}$ & $\begin{array}{l}\mathrm{E}_{3}=0.11 \pm 0.02 \mathrm{MPa}, \mathrm{E}_{1}=1.9 \pm 0.8 \mathrm{MPa}, \\
\mathrm{v}_{21}=\mathrm{v}_{31}=0.0, \mathrm{G}=0.055 \mathrm{MPa}, \\
\mathrm{k}_{0}=14.1 \pm 0.5 \times 10^{-15} \mathrm{~m}^{4} / \mathrm{Ns}, \\
\text { strain-dependance factor }=4.7 \pm 0.9 \text { equilibrium } \\
\text { state }\end{array}$ \\
\hline $\begin{array}{l}\text { Cohen, } \\
\text { et al. }(1998) \\
{[17]}\end{array}$ & calves & 4 months $^{\mathrm{a}}$ & distal ulnae & $\begin{array}{l}\text { unconfined/confined } \\
\text { compression stress } \\
\text { relaxation tests }\end{array}$ & $\begin{array}{l}\text { transversely } \\
\text { isotropic } \\
\text { biphasic; } \\
\text { isotropic }\end{array}$ & $\begin{array}{l}\mathrm{E}_{3}=0.47 \pm 0.11 \mathrm{MPa}, \mathrm{E}_{1}=4.55 \pm 1.21 \mathrm{MPa} \\
\mathrm{v}_{21}=0.30 \pm 0.20, \mathrm{v}_{31}=0.0 \\
\mathrm{k}_{3}=3.4 \pm 1.6 \times 10^{-15} \mathrm{~m}^{4} / \mathrm{Ns} \\
\mathrm{k}_{1}=5.0 \pm 1.8 \times 10^{-15} \mathrm{~m}^{4} / \mathrm{Ns} \text { (biphasic); } \\
\mathrm{E}=1.08 \mathrm{Mpa}, \mathrm{v}=0.0, \mathrm{k}=15.5 \times 10^{-15} \mathrm{~m}^{4} / \mathrm{Ns} \\
\text { (isotropic) equilibrium state }\end{array}$ \\
\hline $\begin{array}{l}\text { Tutorino, } \\
\text { et al. }(2001) \\
\text { [13] }\end{array}$ & calves & 4 - 5 months $^{\mathrm{b}}$ & proximal tibia & $\begin{array}{l}\text { compression stress } \\
\text { relaxation tests }\end{array}$ & & $\begin{array}{l}\mathrm{E}=0.76 \pm 0.24 \mathrm{MPa} \text { (equilibrium modulus); } \\
\mathrm{E}=1.29 \pm 0.51 \mathrm{MPa} \\
\text { (toe modulus, } 15 \% \text { - } 20 \% \text { strain) }\end{array}$ \\
\hline
\end{tabular}

${ }^{\mathrm{a}}$ Immature stage: chondroepiphysis still present. Results are for columnar regions; ${ }^{\mathrm{b}}$ Late stage of development: secondary center of ossification completed.

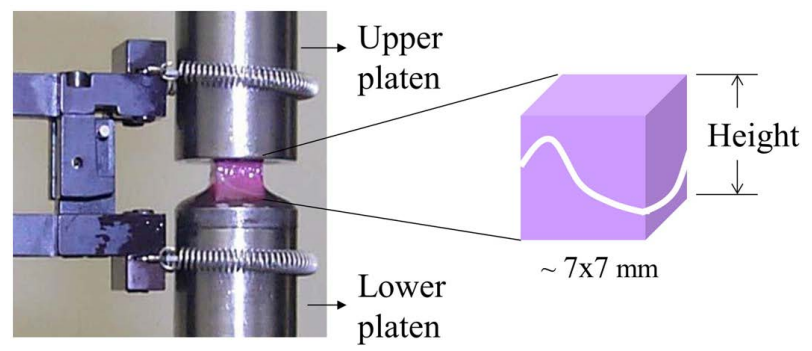

Figure 2. Testing set-up of previous compression experiments. Bone-growth plate-bone sample is held between the compression platens and the upper platen is lowered at a rate of $0.055 \mathrm{~mm} / \mathrm{min}$.

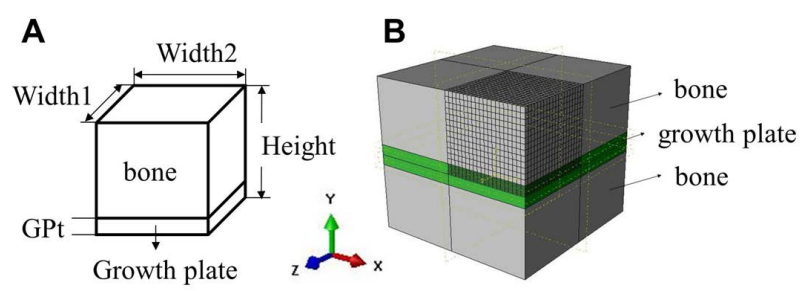

Figure 3. (A) Schematic of the experimental sample; (B) Typical finite element model. At least 6069 C3D8R type of elements were used. The XY, YZ and XZ planes are axes of symmetry.

\subsection{Inverse Parametric Finite Element Analyses}

Twelve FE models were built based on the actual dimensions of individual sample (Table 2) using ABAQUS (Providence, Rhode Island). The FE model shown in Figure $3 \mathrm{~B}, 1 / 8$ of an actual sample, shows the characterization of the bulk response of the growth plate from the uniaxial compression experiments. A homogeneous isotropic linear elastic material was used to model the growth plate cartilage. The Poisson's ratio of the growth plate was chosen to be 0.45 in this study.
Table 2. Geometrical information of 12 specimens utilized in this study.

\begin{tabular}{cccccc}
\hline Specimen & $\begin{array}{c}\text { Width1 } \\
(\mathbf{m m})\end{array}$ & $\begin{array}{c}\text { Width2 } \\
(\mathbf{m m})\end{array}$ & $\begin{array}{c}\text { Height(H) } \\
(\mathbf{m m})\end{array}$ & $\begin{array}{c}\text { GPt } \\
(\mathbf{m m})\end{array}$ & $\mathbf{R Z \%}$ \\
\hline$\# 1$ & 7.085 & 6.746 & 7.310 & 0.514 & 32 \\
$\# 2$ & 6.796 & 6.737 & 6.567 & 0.664 & 29 \\
$\# 3$ & 6.626 & 6.761 & 8.385 & 0.560 & 56 \\
$\# 4$ & 6.833 & 6.746 & 4.938 & 0.798 & 18 \\
$\# 5$ & 6.781 & 6.661 & 5.085 & 0.632 & 32 \\
$\# 6$ & 7.691 & 6.705 & 4.931 & 0.619 & 25 \\
$\# 7$ & 6.749 & 6.870 & 5.462 & 0.656 & 20 \\
$\# 8$ & 6.819 & 6.792 & 6.145 & 0.764 & 36 \\
$\# 9$ & 6.905 & 6.828 & 5.618 & 0.724 & 27 \\
$\# 10$ & 6.809 & 6.755 & 4.530 & 0.676 & 27 \\
$\# 11$ & 6.714 & 6.612 & 6.289 & 0.601 & 40 \\
$\# 12$ & 6.817 & 6.754 & 6.124 & 0.816 & 34 \\
\hline
\end{tabular}

Based on material parameters used in previous studies, the elastic modulus and Poisson's ratio of trabecular bone was $100 \mathrm{MPa}$ and 0.3, respectively [14]. Symmetric boundary conditions were implemented in the front, left and bottom surfaces of the model. Displacement control was applied incrementally on the top surface of the model until 20\% strain level of the growth plate layer was reached. A mesh convergence study was also performed in order to determine an appropriate element size, especially for the growth plate region. Furthermore, in order to eliminate volumetric locking issues due to the near incompressibility of growth plate, 8 node solid elements with reduced integration formulation were used (C3D8R). Parametric studies were then conducted for each sample where the elastic modulus of the growth plate in the FE model was altered systematically in order to find the optimal fit between the FE simulated and experimental 
stress-strain curves.

A nominal stress was obtained by dividing the total reaction force, which is sum of the reaction forces at the base, by the initial cross-sectional area. Using this nominal stress, an intrinsic elastic modulus for the growth plate cartilage FE experiment $\left(E_{I N}\right)$ was calculated. The equilibrium modulus, which can be considered as the elastic modulus at $20 \%$ strain level, was calculated for each specimen by dividing the equilibrium stress by the grip-to-grip strain and reported previously [13]. The slopes of these twelve stress-strain curves, which reflect the extrinsic elastic moduli of these samples $\left(E_{E X}\right)$, were obtained by curve fitting a straight line to the data between 0 and 20\% strain using Matlab (Natick, MA).

Based on these parametric studies, elastic modulus values for the growth plate cartilage $\left(E_{I N}\right)$ that provided the optimal fit between experimental and FE stress-strain curves were determined. The ratio of $E_{I N}$ and $E_{E X}$ was also calculated.

\subsubsection{Simulation of Previous Uniaxial Compression Experiments of Macroscopic Samples}

When the FE models were constructed, there was no detailed information regarding the internal geometry of these twelve samples. Therefore, the growth plate layer in the FE models was modeled to be flat. However, in order to investigate the impact of topography in the growth plate geometry in our calculated $E_{I N}$, we constructed additional FE models with different geometrical structures of the growth plate layer, while maintaining all other dimensions and the underlying material properties the same. For comparison with the flat shaped model, we followed a previous study [15] and utilized " $n$ " and "m" shaped growth plate layers (Figures 4A and B), which represent in a simplified manner the shape of secondary mammi-

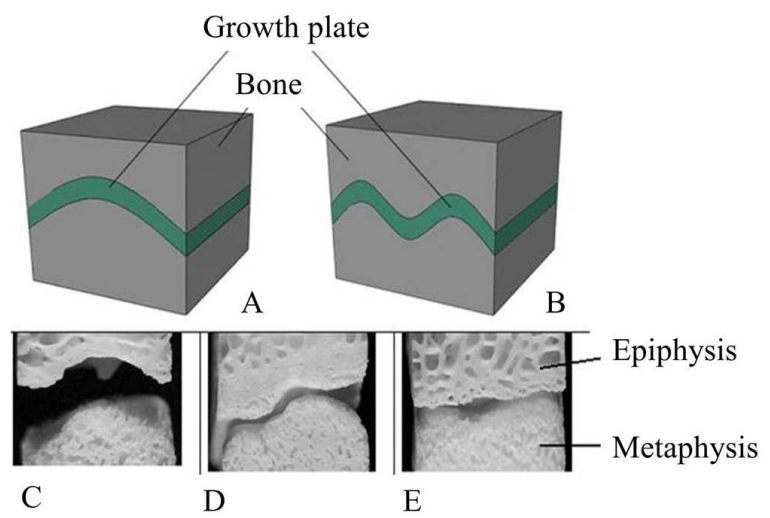

Figure 4. Topography of the growth plate layer. (A) "n" shaped; (B) " $\mathrm{m}$ " shaped, representing the varied interdigitations observed in the test specimens. Photographs of specimens show the secondary mamillary processes after removal of the cartilage and marrow with a solution of bleach (C through E, epiphyseal bone on top). lary processes found in these tested specimens from the cow (Figures 4C-E). The amplitudes of the modeled $n$ and $\mathrm{m}$ shapes were twice the growth plate thickness values. We also computed the dependence of the difference between the $E_{E X}$ and $E_{I N}$ on the shape of growth plate.

\subsubsection{Bilayered Growth Plate Model}

In order to explore the influence of assuming homogeneity of Young's modulus through the growth plate thickness on the $E_{I N}$ values derived from the twelve FE models, we constructed a FE model of one sample (\#12) using a quarter model of the bone/growth plate/bone structure. The growth plate layer was assumed to be flat, but partitioned into two sections, consisting of the reserve zone and proliferative/hypertrophic zone. The proportion of the overall growth plate thickness or height occupied by the reserve zone (RZ\%) was previously determined for each sample by averaging measurements on all four faces of each sample (Table 2). Since the reserve zone has been found to be nearly twice as stiff as the proliferative/hypertrophic zone in the loading direction [16,17], the elastic modulus of the reserve zone was constrained to be twice of the proliferative/hypertrophic zone, whilst the Poisson's ratio kept the same as in previous models.

\section{Results}

\subsection{Extrinsic Elastic Modulus of the Growth Plate Cartilage from Experiments}

Twelve bovine growth plate samples were tested in quasi-static compression [13] and information pertaining to these samples is presented in Table 2. Results of the parametric study for one bovine growth plate sample are shown in Figure 5. The overall height of the compression samples averaged $5.95 \pm 1.11$ (SD) $\mathrm{mm}$ and the growth plate height $(\mathrm{GPt})$ was $0.67 \pm 0.09$ (SD) $\mathrm{mm}$ on average. The average proportion of the reserve zone (RZ\%) was $31.33 \% \pm 10.00 \%$ (SD). The extrinsic elastic modulus for these samples is $1.11 \pm 0.40$ (STD) MPa.

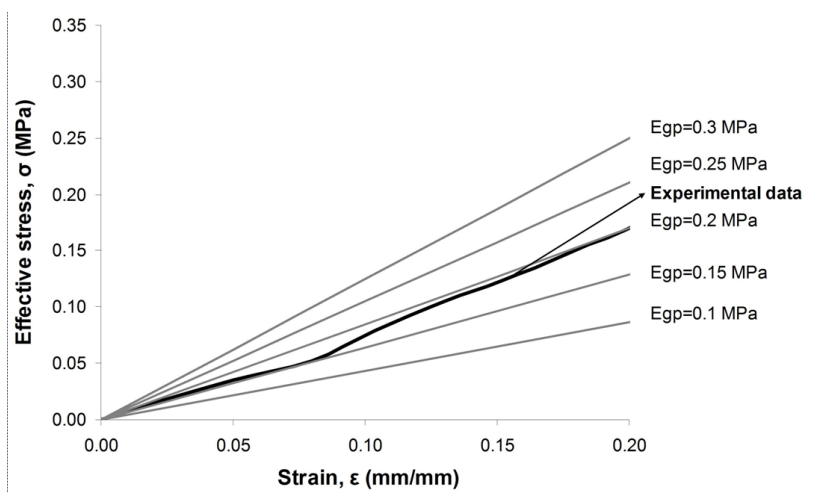

Figure 5. Parametric finite element compression simulation of one sample. 


\subsection{Intrinsic Elastic Modulus of the Growth Plate Cartilage from Inverse FE Experiments}

For each experimental sample, we constructed an individual FE model to obtain the underlying $E_{I N}$. Table 3 shows the $E_{E X}$ and $E_{I N}$ for all twelve samples that underwent the inverse $\mathrm{FE}$ analyses. The resulting average $E_{I N}$ for the twelve bovine growth plate samples is $0.36 \pm 0.15$ $\mathrm{MPa}$, which is about $30 \%$ of the $E_{E X}$ computed directly from the experiments. The extrinsic compressive elastic modulus, $E_{E X}$, was between 2 and 4.4 times the value of the intrinsic modulus, $E_{I N}$.

\subsubsection{Different Growth Plate Topography}

We also studied the impact of the topography of the secondary mammillary processes on the difference between $E_{E X}$ and $E_{I N}$. As the results in Table 4 indicate, the shape of the growth plate does indeed influence the overall mechanical response of the bone/growth plate/bone sample under uniaxial compression and the material parameters. According to these results, the larger the ratio of $\mathrm{GPt} /$ height of the sample, the greater the impact of growth plate topography on the difference between $E_{E X}$ and $E_{I N}$. Based on the results of the three idealized shapes, it shows that the "m" shaped growth plate layer can lead to a greater difference between $E_{E X}$ and $E_{I N}$ for the same sample compared to the " $n$ " shaped one. For example,

Table 3. Summarized results for twelve specimens.

\begin{tabular}{ccccc}
\hline Specimen & $\boldsymbol{E}_{\boldsymbol{E X}}$ (Mpa) & $\boldsymbol{E}_{\boldsymbol{I N}}(\mathbf{M p a})$ & $\boldsymbol{E}_{\boldsymbol{E X}} / \boldsymbol{E}_{\boldsymbol{I N}}$ & $\mathbf{G P t} / \mathbf{H}$ \\
\hline \#1 & 1.3744 & 0.5564 & 2.47 & 0.07 \\
\#2 & 0.5154 & 0.1778 & 2.90 & 0.10 \\
\#3 & 0.9001 & 0.4487 & 2.01 & 0.07 \\
\#4 & 1.2280 & 0.3357 & 3.66 & 0.16 \\
\#5 & 0.6696 & 0.1758 & 3.81 & 0.12 \\
\#6 & 1.2107 & 0.2728 & 4.44 & 0.13 \\
\#7 & 1.9816 & 0.6284 & 3.15 & 0.12 \\
\#8 & 1.3338 & 0.4697 & 2.84 & 0.12 \\
\#9 & 1.1370 & 0.3453 & 3.29 & 0.13 \\
\#10 & 0.8402 & 0.1980 & 4.24 & 0.15 \\
\#11 & 0.7555 & 0.2549 & 2.96 & 0.10 \\
\#12 & 1.3486 & 0.4801 & 2.81 & 0.13 \\
AVERAGE & 1.1079 & 0.3620 & 3.2150 & 0.1167 \\
STDEV & 0.3990 & 0.1531 & 0.7128 & 0.0277 \\
\hline
\end{tabular}

Table 4. Influence of growth plate layer topography on the ratio $\left(E_{E X} / E_{I N}\right)$.

\begin{tabular}{ccccccc}
\hline Sample & $\begin{array}{c}\text { GPt } \\
(\mathbf{m m})\end{array}$ & GPt/H & $\begin{array}{c}\boldsymbol{E}_{I N} \\
\text { (Mpa) }\end{array}$ & flat & $\begin{array}{c}\text { “n” } \\
\text { shaped }\end{array}$ & $\begin{array}{c}\text { “m” } \\
\text { shaped }\end{array}$ \\
\hline 1 & 0.514 & 0.07 & 0.362 & 2.79 & $\begin{array}{c}2.79 \\
(0.0 \%)\end{array}$ & $\begin{array}{c}2.91 \\
(4.3 \%)\end{array}$ \\
2 & 0.664 & 0.1 & 0.362 & 2.76 & $\begin{array}{c}2.82 \\
(2.2 \%)\end{array}$ & $\begin{array}{c}2.98 \\
(8.0 \%)\end{array}$ \\
& & & & & $\begin{array}{c}2.76 \\
2.95\end{array}$ \\
& 0.816 & 0.13 & 0.362 & 2.67 & $(3.4 \%)$ & $(10.5 \%)$ \\
\hline
\end{tabular}

the value for $E_{I N}$ for the "m" shaped growth plate can be $10.5 \%$ more than for a flat growth plate of the same height.

\subsubsection{Bilayered Growth Plate Model}

Recognizing that the growth plate is composed of microscopic features with significant differences between the histological zones, we aimed to refine our model by partitioning the growth plate into two zones. The reserve zone was $34 \%$ of the overall height of the growth plate and the elastic modulus, $E_{R Z}$, was constrained to be twice that of the columnar and hypertrophic zones, modeled by a single layer, $E_{P+H}$. Using this approach, compressive elastic moduli of the bovine reserve and proliferative/ hypertrophic zones were determined to be $0.74 \mathrm{MPa}$ and $0.37 \mathrm{MPa}$, respectively.

\section{Discussion}

In this study, we simulated compression tests on macroscopic bone/growth plate/bone samples to extract the intrinsic elastic modulus of bovine growth plate cartilage using parametric inverse FE analyses. The extrinsic elastic modulus was approximately 3 times greater than the estimated intrinsic growth plate cartilage elastic modulus. However, since we found a strong correlation between $E_{I N} / E_{E X}$ and sample height: $E_{I N} / E_{E X}=-0.071+0.067 *$ height $\left(\mathrm{R}^{2}=0.95, \mathrm{p}<0.0001\right)$ further discussion is warranted.

\subsection{Triaxial Stress State during Growth Plate Compression}

The intrinsic elastic modulus values we computed are in general lower than those previously reported $[1,18]$. Part of the reason for this discrepancy is that the triaxial state of stress present in a thin layer of soft material sandwiched between stiffer materials is usually not accounted for when the elastic modulus is calculated from uniaxial compression tests. This triaxial state of stress is known to exist in thin flat test samples that are constrained from lateral displacement at the surfaces perpendicular to the applied compression direction. Such conditions may be intentionally induced in order to examine a material's behavior under hydrostatic stress, i.e. the pancake test [19]. Similarly, in the case of the growth plate of a long bone in which the original fully cartilaginous epiphysis (chondroepiphysis) has been transformed into bone just a thin layer of growth cartilage $(1-2 \mathrm{~mm})$ is left between the epiphyseal and metaphyseal bone on either side. This type of stress is also present in experiments where a thin layer of cartilage has been completely isolated from the bone and subjected to confined or unconfined compression unless friction at the platen surfaces can be significantly reduced. It is also reasonable to suggest that the com- 
pression of macroscopic test bone-growth plate-bone samples mimic the in vivo physiological state of stress in which the cartilage surface at the bone borders are partially constrained by the compatibility displacement conditions and are not free to expand.

\subsection{Relevance to Bone Growth Theories}

Hydrostatic stress state is thought to maintain cartilage and octahedral shear stress is thought to lead to calcification $[8,9]$. Therefore, we computed the hydrostatic and octahedral shear stress in the middle of the growth plate, i.e. halfway between the epiphyseal and metaphyseal borders (Figure 6). The absolute value of hydrostatic stress is an order of magnitude greater than the octahedral shear stress suggesting that the center of the growth plate is experiencing a near hydrostatic stress state. Both stresses exhibited dependence on the location from the free surfaces of the specimen, where their absolute magnitudes approached a similar value at the outer edge. For the center of the growth plate, where there was no outer edge effect, the hydrostatic stress and octahedral shear stress remained nearly constant (Figures 7 and 8). In order to compare the influence of different growth plate geometries, similar plots were then created for two other models with "n" and "m" shaped growth plate layers to show the stress distributions. As results show in Figure 8, they all followed a similar pattern with little or no difference in the hydrostatic or octahedral shear stress at the specimen center among these growth plates with differing geometry. Away from the center, the differences were at most about $20 \%$ for the hydrostatic and octahedral shear stress, relative to the flat growth plate geometry. Since the samples were cut from a large animal growth plate, they contain free surfaces, which are not present in the in situ state. Thus the variation in the state of stress from the center to the edge as determined for the tested specimens is not representative of what would be expected in situ or in vivo in a larger animal such as the cow, where

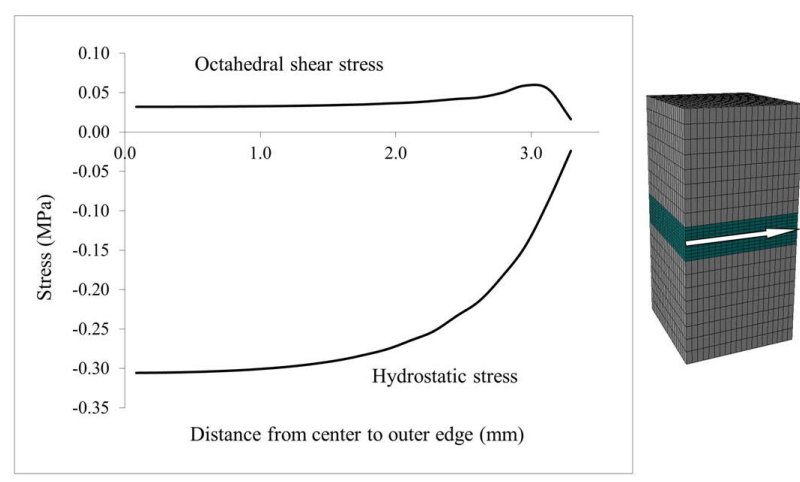

Figure 6. Hydrostatic and octahedral shear stresses in the middle of the growth plate (arrow indicates towards the outer edge).

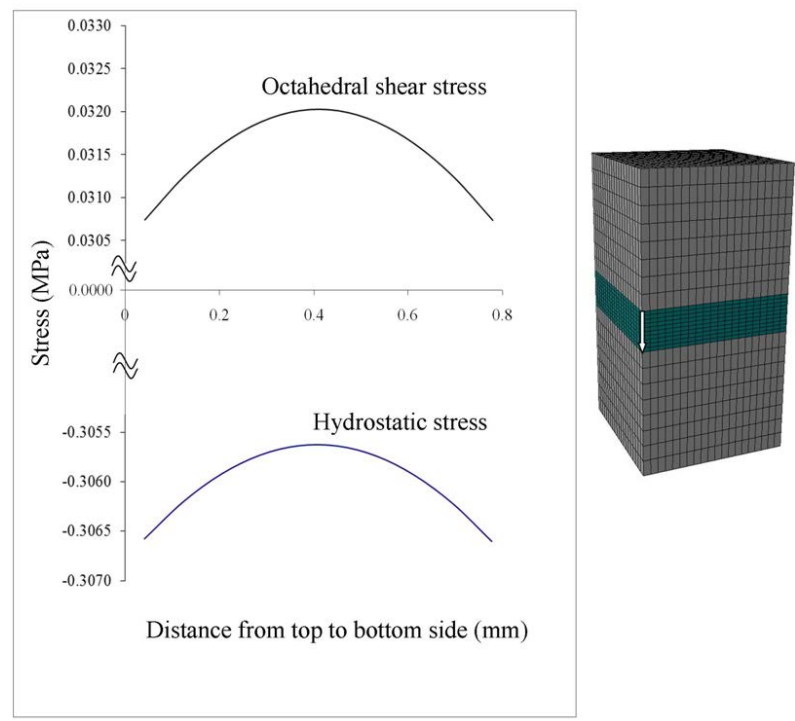

Figure 7. Stress distribution of the center column of elements in the growth plate (arrow runs from epiphyseal to metaphyseal side).

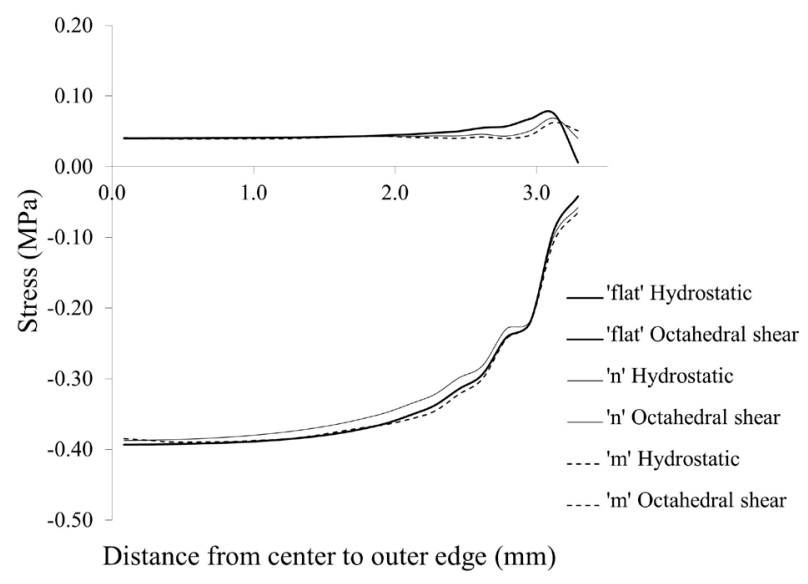

Figure 8. Influence of growth plate geometry on middle layer stresses in a different sample from that shown in Figure 6.

free surfaces only exist at the perichondrium.

On the other hand, it should be noted that there is a close correspondence between the specimen dimensions of these macroscopic growth plate samples, which were cut from the growth plate of a large mammal (cow), and the dimensions of the entire growth plate of a long bone in a small mammal such as a rat. Likewise the topography of the secondary mammillary processes in these specimens obtained from the cow corresponds to that of the primary mammillary processes in a small mammal such as rat. Thus the state of stress determined for the extracted cow samples may be representative of the state of stress in the entire growth plate of a small animal. This suggests then, if hydrostatic stress state maintains cartilage and octahedral shear stress causes cartilage degrada- 
tion and accelerates ossification, that compression would promote bone formation near the external surfaces of the growth plate more so than at the center. Perhaps this explains the topography of the proximal tibial primary mammillary processes which, when viewed in a coronal slice, exhibit some convex curvatures at the medial and lateral borders (Figure 1A). This is similar in shape to what is seen in the rat proximal tibial growth plate in the frontal plane (coronal section) [20] and also similar to the m-shaped secondary mammillary process modeled in this study for macroscopic samples. The m-shaped primary mammillary processes found at the periphery of the growth plate in the rat and cow suggest that octahedral shear stress accelerates bone growth/formation and hydrostatic stress (near the center) retards growth by maintaining cartilage.

Elastic moduli determined for each of the two sections of the growth plate from the bilayer FE model are comparable in magnitude to those previously reported [16,17]. However, direct comparison with other studies is difficult due to the use of different animal species [16], anatomic regions, stage of development [17], test methodology and material property assumptions [17]. One study [17] conducted confined and unconfined compression of $2 \mathrm{~mm}$ thick cartilage discs prepared from 5-month-old calf distal ulnar growth plates by cutting the cartilage at the metaphyseal border. Since the epiphyseal side in this location consisted of a chondroepiphysis the other end could be trimmed to leave a disk consisting only of cartilage [17]. Although it is not clear whether this could be done without damaging the hypertrophic layer, given the undulations of the mammillary processes, this would not at all be possible to do in a growth plate that is fully developed and consists of bone on both sides of the growth plate, unless the thickness of the cartilage is sufficient and the mammilary processes are small. Interestingly, in the case of the rat, a careful microCT study has shown that the normal time course of growth plate closure by bone bridging in the proximal tibial in the rat occurs first around the periphery and then progresses toward the center [20]. This sequence may also be related to the nature of the stress distribution at the time of growth plate closure when the cartilage cells reach apoptosis. Perhaps octahedral shear stress accelerates the formation bone bridges while hydrostatic stress preserves cartilage.

\subsection{Limitations and Underlying Assumptions}

We recognize that there are limitations to our approach. In our model, we only considered the condition of slowly ramped compressive loading along an axis perpendicular to the main plate direction and ignored fluid related contributions to the stress. In addition, we analyzed experimental data in which a $20 \%$ grip-grip strain level was slowly applied and maintained until the nominal stress reached equilibrium. This allowed us to consider the bovine growth plate as a linearly elastic material, although we are aware of the various nonlinear material models that have been considered $[8,14]$. There are various types of material models which have been used for the growth plate when it comes to FE modeling, such as nonlinear biphasic models [21], linear biphasic poroelastic models [22], transversely isotropic biphasic models [17]. Compared to these, a linear elastic model is still very attractive and sufficient enough to be used to describe the basic mechanical behavior of the growth plate under uniaxial compression $[8,14]$, at least to relatively lower strain levels and slowly applied compression. Another important parameter in our modeling is the Poisson's ratio of the growth plate, which prior studies report anywhere from $\leq 0.1$ [1] to slightly less than 0.5 [23]. In this study, 0.45 was used based on the assumption of nearly incompressible mechanical response of the growth plate due to the high cellular content.

A major limitation to our study was the lack of full geometrical information of the samples. The topography of the growth plate cartilage can be very different from one specimen to another (Figures 4C-E). Due to lack of information regarding the internal topography of the growth plate cartilage layer, the models did not fully take into account of the undulations of the cartilage layer. The models assumed the growth plate layer to be flat and perpendicular to the force and height of the specimen. We found a strong linear dependence of $E_{I N} / E_{E X}$ on height $\left(\mathrm{R}^{2}=0.95, \mathrm{p}<0.0001\right)$. The explanation for this is that the model assumed the growth plate to be flat and perpendicular to the force and height of the specimen.

Two simplified mildly undulating shapes of the growth plate layer were modeled to compare with the flat shape. However, the actual mamillary undulations in the samples were more severe than the modeled shapes and encompassed the entire specimen height and slopes changes can be aggressive. The more sloped it is (i.e. higher specimen) the more shear the growth plate cartilage is exposed to. However, the models used to estimate the intrinsic modulus consisted of a growth plate, which is primarily exposed to compression. Therefore, the intrinsic modulus extracted from this model for these specimens is more likely that of the modulus in combination of shear and compression, which explains the dependence of $E_{I N} /$ $E_{E X}$ on specimen height. Therefore, the unique topography of the growth plate layer in each sample should be taken into account while using data from such experiments.

Secondly, the zone of calcified cartilage/primary spongiosa, which lies between growth plate and metaphyseal bone, is a region of likely increased compliance compared to the more mature secondary spongiosa and this 
was not taken into account in the models. Since this zone has a thickness comparable to that of the growth plate cartilage, including this in the model may alter the calculated intrinsic modulus of the less mineralized proliferative and hypertrophic zones and reserve zones. One could argue that the zone of provisional calcification or primary spongiosa should be considered as part of the growth plate proper, though most biomechanical studies to date have not considered this.

\section{Conclusions and Summary}

In summary, we report the state of stress in the growth plate cartilage of block-shaped samples containing bone and growth-plate with $7 \times 7 \mathrm{~mm}$ cross sections, excised from the proximal tibias of 5 month old calves. We utilized FE analysis to model the sample structure and to estimate the intrinsic elastic modulus of the growth plate cartilage by simulating and matching the uniaxial compression tests. The stress state in the growth plate was triaxial, nonuniform across the cross section, and predominantly hydrostatic over most of the central region but became an equal mixture of octahedral shear stress and hydrostatic stress near the external surface of the bone samples.

Limitations of the current model include the relatively flat approximation of the mamillary processes in the model compared to the more extreme undulations of the experimental specimens and the exclusion of the zone of provisional calcification. The latter may function as a mechanical buffer zone between the resilient growth plate cartilage and the comparatively stiffer secondary spongiosa. We believe that further improvement of these models will lead us to a better understanding of how macroscopic loads are experienced by chondrocytes at the microscopic level.

\section{REFERENCES}

[1] I. Villemure and I. A. F. Stokes, "Growth Plate Mechanics and Mechanobiology. A Survey of Present Understanding," Journal of Biomechanics, Vol. 42, No. 12, 2009, pp. 1793-1803. http://dx.doi.org/10.1016/j.jbiomech.2009.05.021

[2] J. P. Iannotti, S. Goldstein, J. Kuhn, L. Lipiello, F. S. Kaplan and D. J. Zaleske, "The Formation and Growth of Skeletal Tissue,” In: J. A. Buckwalter, T. A. Einhorn, S. R. Simon, et al., Eds., Orthopaedic Basic Science. Biology and Biomechanics of the Musculoskeletal System Basic Science. Biology and Biomechanics of the Musculoskeletal System, American Academy of Orthopaedic Surgeons, 2000, pp. 77-109.

[3] I. A. F. Stokes, "Mechanical Effects on Skeletal Growth," Journal of Musculoskeletal and Neuronal Interactions, Vol. 2, No. 3, 2002, pp. 277-280.

[4] R. Hall, “A Mathematical Model for Longitudinal Bone
Growth,” Master Thesis, Oxford University, Oxford, 2000.

[5] C. Hueter, "Anatomische Studien an den Extremitaetengelenken Neugeborener und Erwachsener," Archiv für Pathologische Anatomie und Physiologie und für Klinische Medicin, Vol. 25, No. 5-6, 1862, pp. 572-599.

[6] R. Volkmann, "Verletzungen und Kankenheiten der Bewegungsorgane,” In: F. R. von Pitha and T. Billroth, Eds., Handbuch der Allgemeinen und Speciellen Chirurgie $B d$ II Teil II, Ferdinand Enke, Stuttgart, 1882.

[7] H. M. Frost, "Defining Osteopenias and Osteoporoses: Another View (with Insights from a New Paradigm)," Bone, Vol. 20, No. 5, 1997, pp. 385-391. http://dx.doi.org/10.1016/S8756-3282(97)00019-7

[8] D. R. Carter and M. Wong, "Modelling Cartilage Mechanobiology," Philosophical Transactions of the Royal Society of London Series B-Biological Sciences, Vol. 358, No. 1437, 2003, pp. 1461-1471. http://dx.doi.org/10.1098/rstb.2003.1346

[9] D. R. Carter and M. Wong, "The Role of Mechanical Loading Histories in the Development of Diarthrodial Joints,” Journal of Orthopaedic Research, Vol. 6, No. 6, 1988, pp. 804-816. http://dx.doi.org/10.1002/jor.1100060604

[10] H. M. Frost, "Skeletal Structural Adaptations to Mechanical Usage (SATMU). 3. The Hyaline Cartilage Modeling Problem. Anatomical Record," The Anatomical Record, Vol. 226, No. 4, 1990, pp. 423-432. http://dx.doi.org/10.1002/ar.1092260404

[11] J. J. Mao and H. D. Nah, "Growth and Development: Hereditary and Mechanical Modulations," American Journal of Orthodontics and Dentofacial Orthopedics, Vol. 125, No. 6, 2004, pp. 676-689.

http://dx.doi.org/10.1016/j.ajodo.2003.08.024

[12] G. H. Thompson and J. R. Carter, "Late-Onset Tibia Vara (Blount's Disease)—Current Concepts,” Clinical Orthopaedics and Related Research, Vol. 255, 1990, pp. 24-35.

[13] J. C. Tutorino, Z. G. Khubchandani, J. L. Williams, C. M. Cobb and T. L. Schmidt, "Can The Epiphyseal Growth Plate be Injured in Compression?” In: B. S. Trippel, Ed., Transactions of the 47th Annual Meeting of the Orthopaedic Research Society, Vol. 26, Orthopaedic Research Society, Rosemont, IL, 2001, p. 353.

[14] S. Piszczatowski, "Material Aspects of Growth Plate Modelling Using Carter's and Stokes's Approaches,” Acta of Bioengineering and Biomechanics, Vol. 13, No. 3, 2011, pp. 3-14.

[15] S. Piszczatowski, "Geometrical Aspects of Growth Plate Modelling Using Carter's and Stokes’s Approaches,” Acta of Bioengineering and Biomechanics, Vol. 14, No. 1, 2012, pp. 93-106.

[16] K. Sergerie, M. O. Lacoursiere, M. Levesque and I. Villemure, "Mechanical Properties of the Porcine Growth Plate and Its Three Zones from Unconfined Compression Tests,” Journal of Biomechanics, Vol. 42, No. 4, 2009, pp. 510-516. http://dx.doi.org/10.1016/j.jbiomech.2008.11.026

[17] B. Cohen, W. M. Lai and V. C. Mow, “A Transversely 
Isotropic Biphasic Model for Unconfined Compression of Growth Plate and Chondroepiphysis," Journal of Biomechanical Engineering-Transactions of the ASME, Vol. 120, No. 4, 1998, pp. 491-496.

http://dx.doi.org/10.1115/1.2798019

[18] P. Radhakrishnan, N. T. Lewis and J. J. Mao, “ZoneSpecific Micromechanical Properties of the Extracellular Matrices of Growth Plate Cartilage,” Annals of Biomedical Engineering, Vol. 32, No. 2, 2004, pp. 284-291. http://dx.doi.org/10.1023/B:ABME.0000012748.41851.b 4

[19] G. A. Laughlin, J. L. Williams and J. D. Eick, “The Influence of System Compliance and Sample Geometry on Composite Polymerization Shrinkage Stress,” Journal of Biomedical Materials Research, Vol. 63, No. 5, 2002, pp. 671-678. http://dx.doi.org/10.1002/jbm.10386

[20] E. A. Martin, E. L. Ritman and R. T. Turner, "Time Course of Epiphyseal Growth Plate Fusion in Rat Tibiae," Bone, Vol. 32, No. 3, 2003, pp. 261-267. http://dx.doi.org/10.1016/S8756-3282(02)00983-3

[21] B. Cohen, G. S. Chorney, D. P. Phillips, H. M. Dick and
V. C. Mow, "Compressive Stress-Relaxation Behavior of Bovine Growth-Plate May Be Described by the Nonlinear Biphasic Theory,” Journal of Orthopaedic Research, Vol. 12, No. 6, 1994, pp. 804-813. http://dx.doi.org/10.1002/jor.1100120608

[22] V. C. Mow, S. C. Kuei, W. M. Lai and C. G. Armstrong, "Biphasic Creep and Stress-Relaxation of Articular-Cartilage in Compression-Theory and Experiments," Journal of Biomechanical Engineering, Vol. 102, No. 1, 1980, pp. 73-84. http://dx.doi.org/10.1115/1.3138202

[23] M. Wong and D. R. Carter, "A Theoretical-Model of Endochondral Ossification and Bone Architectural Construction in Long-Bone Ontogeny," Anatomy and Embryology, Vol. 181, No. 6, 1990, pp. 523-532. http://dx.doi.org/10.1007/BF00174625

[24] F. Barthelat, D. Fonck and A. L. Lerner, "Investigation of the Poroelastic Behavior of the Rabbit Growth Plate Cartilage,” In: V. K. Goel, R. L. Spilker, G. A. Athesian, L. J. Soslowsky, Eds., Proceedings of the 1999 Bioengineering Conference, BED-Vol. 42, ASME, New York, NY, 1999, pp. 757-758. 